\title{
Monte Carlo Analysis of Impact Ionization in Isolated-Gate InAs/AlSb High Electron Mobility Transistors
}

\author{
B.G. Vasallo ${ }^{a}$, H. Rodilla ${ }^{a}$, T. GonzÁlez ${ }^{a}$, E. Lefebvre ${ }^{b}$, G. Moschetti $^{c}$, \\ J. GRAHN ${ }^{c}$ AND J. MATEOS ${ }^{a}$ \\ ${ }^{a}$ Dpto. Física Aplicada, Universidad de Salamanca, Plaza de la Merced s/n, 37008 Salamanca, Spain \\ ${ }^{b}$ Osram Semiconductor, Regensburg, Germany \\ ${ }^{c}$ Department of Microtechnology and Nanoscience - MC2, Chalmers, University of Technology \\ SE-412 96 Göteborg, Sweden
}

\begin{abstract}
We perform a physical analysis of the kink effect in InAs/AlSb high electron mobility transistors by means of a semiclassical 2D ensemble Monte Carlo simulator. Due to the small bandgap of InAs, InAs/AlSb high electron mobility transistors are very susceptible to suffer from impact ionization processes, with the subsequent hole transport through the structure, both implicated in the kink effect. When the drain-to-source voltage $V_{\mathrm{DS}}$ is high enough for the onset of impact ionization, holes generated tend to pile up at the gate-drain side of the buffer. This occurs due to the valence-band energy barrier between the buffer and the channel. Because of this accumulation of positive charge, the channel is further opened and the drain current $I_{\mathrm{D}}$ increases, leading to the kink effect in the $I-V$ characteristics.
\end{abstract}

PACS: 85.30.De, 85.30.Tv

\section{Introduction}

Due to the high mobility of electrons in InAs and the excellent electron confinement in the channel, InAs/AlSb heterostructures are being considered to further improve the performance of high electron mobility transistors (HEMTs) for low-power, high-frequency and low-noise applications [1-4]. However, there are some problems to be eliminated, as the kink effect, an anomalous increase of the drain current $I_{\mathrm{D}}$ when increasing the drain-to-source voltage $V_{\mathrm{DS}}$, caused by impact ionization and the subsequent hole dynamics in the structure. Kink effect leads to a reduction in the gain and a rise in the level of noise, thus limiting the utility of these devices for microwave applications. Thus, the development of strategies to reduce the negative consequences of kink effect on the operation of InAs/AlSb HEMTs is indispensable. In order to overcome the appearance of the associated excessive gate leakage current related to impact-ionization generated holes, the conventional Schottky contact has been replaced by an insulated gate by means of a native oxide [5]. Nevertheless, impact ionization events are extremely frequent because of the low band-gap in the channel material, and thus these devices can be much affected by kink effect.

The purpose of this work is to perform an analysis of the physical origin of the kink effect in recessed isolated-gate AlSb/InAs HEMTs. With this aim, we make use of a semi-classical 2D ensemble Monte Carlo (MC) simulator [6] adequately adapted to correctly model InAs/ AlSb heterostructures [7], in which both impact ionization and hole dynamics are included $[8,9]$. The MC technique is the most adequate simulation tool for this

\footnotetext{
* corresponding author; e-mail: bgvasallo@usal.es
}

kind of devices, since electron transport can easily turn into ballistic, or at least quasiballistic, in the channel of the analyzed transistors due to the very high mobility in InAs [7]. The MC method has been also proved to be a very useful tool when dealing with problems in which the understanding of the microscopic behavior of carriers is essential, as when analyzing kink effect phenomena. It allows us to determine the origin and magnitude of the kink effect in terms of internal quantities, such as electron and hole concentrations $[8,9]$.

\section{Physical model}

For the device analysis we make use of an ensemble MC simulator self-consistently coupled with a 2D Poisson solver which incorporates impact ionization and hole dynamics. The simulated device is very similar to the experimental one described in Ref. [4]. The structure under analysis, plotted schematically in Fig. 1, is a $225 \mathrm{~nm}$ T-gate recessed HEMT, fabricated on a heterostructure consisting of a InP substrate (not simulated), a $800 \mathrm{~nm}$ AlSb buffer followed by a $15 \mathrm{~nm}$ thick InAs channel, two layers of AlSb (a $5 \mathrm{~nm}$ spacer and a $10 \mathrm{~nm}$ Schottky layer, with a $\delta$-doped layer between them), and, finally, a $4 \mathrm{~nm}$ thick AlInAs layer and a $5 \mathrm{~nm}$ thick InAs cap layer $\left(N_{\mathrm{D}}=10^{18} \mathrm{~cm}^{-3}\right)$. Details about the electron-transport model in this heterostructure can be found in Ref. [7].

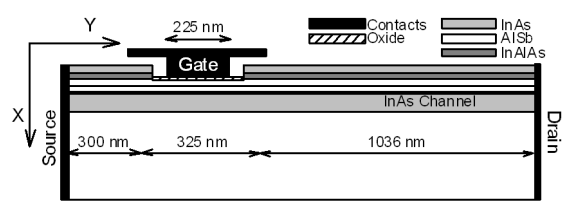

Fig. 1. (a) Schematic drawing of the HEMT topology under analysis. 
Impact ionization, which occurs for electrons in the $\Gamma$ valley of InAs, is included in the MC simulations by using the Keldysh approach [10], where the probability per unit time of having an impact ionization event is $S\left[\left(E-E_{\mathrm{th}}\right) / E_{\mathrm{th}}\right]^{2}$ if $E>E_{\mathrm{th}}$ and 0 otherwise, $E$ being the electron kinetic energy, $E_{\mathrm{th}}$ is the ionization threshold energy and $S$ is a measure of the softness or hardness of the threshold. Impact ionization is negligible for electrons in upper valleys. From each impact ionization occurrence, an electron in the $\Gamma$ valley and a hole in the heavy-hole band emerge, while the electron originating the ionization process remains in the $\Gamma$ valley. Concerning the hole dynamics, a typical spherical and nonparabolic valence band structure is considered, including three sub-bands: heavy- and light-hole bands, degenerated at $\boldsymbol{k}=0$ and characterized by a different curvature in $\boldsymbol{k}$-space, and a third split-off band, in which the band warping is accounted for by the use of approximated overlap functions [11]. Ionized impurity, acoustic, polar and non-polar optical phonon scattering mechanisms are considered for holes [11, 12]. The physical parameters used to model hole transport provide a low-electric-field mobility of $350 \mathrm{~cm}^{2} /(\mathrm{V} \mathrm{s})$ for InAs and $525 \mathrm{~cm}^{2} /(\mathrm{V} \mathrm{s})$ for AlSb, both similar to the respective experimental values [13]. In addition, for a proper analysis of kink effect it is necessary to take into account the hole recombination [8]. To this end, we used a simple model in which this mechanism is considered to occur with a characteristic time $\tau_{\text {rec }}$ (i.e., with a probability $\left.1 / \tau_{\text {rec }}\right) . S, E_{\text {th }}$ and $\tau_{\text {rec }}$ are taken as adjustable parameters to adequately reproduce the experimental static $I-V$ characteristics, shown in Sect. 3.

\section{Results}

Figure 2 shows the simulated (a) intrinsic and (b) extrinsic $I-V$ characteristics of the InAs/AlSb HEMT with and without impact ionization. The experimental $I-V$ curves taken from [4] have been included in Fig. 2b. In order to perform the comparison of the measured results (extrinsic) with those obtained from the simulation (intrinsic), it is necessary to include, in a postprocessing stage, the parasitic elements that are not considered in the intrinsic MC simulation [14]. Thus, drain and source parasitic resistances associated with the contact metallization and part of the ohmic regions not included in the simulation domain have been incorporated into the original $\mathrm{MC}$ results, with the best fit being obtained for $R_{\mathrm{S}}=0.13 \Omega \mathrm{mm}$ and $R_{\mathrm{D}}=0.38 \Omega \mathrm{mm}$. An excellent agreement between experimental and simulated results is reached (Fig. 2b) when considering impact ionization in the simulations with values for the involved parameters of $S=10^{12} \mathrm{~s}^{-1}, E_{\mathrm{th}}=0.41 \mathrm{~V}$ and $\tau_{\text {rec }}=0.5 \mathrm{~ns}$. A notable increase of $I_{\mathrm{D}}$ takes place starting from a value of $V_{\mathrm{DS}}$ high enough so that the electron energy is sufficient for the onset of impact ionization. In contrast, in the absence of impact ionization the output curves are fitted just up to $V_{\mathrm{DS}}=0.2 \mathrm{~V}$.

The increase of $I_{\mathrm{D}}$ for a fixed $V_{\mathrm{DS}}$ due to impact ionization and the appearance of holes grows with $V_{\mathrm{GS}}$. This

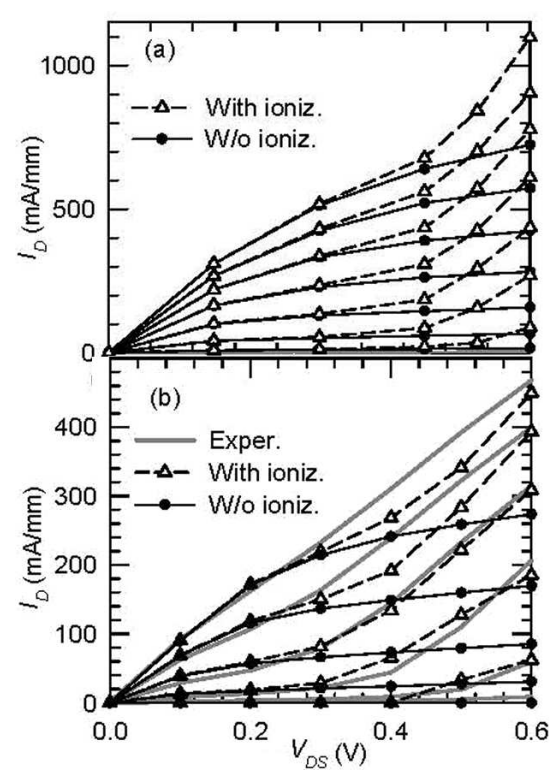

Fig. 2. $\mathrm{MC}$ (a) intrinsic and (b) extrinsic $I-V$ curves in presence and absence of impact ionization, being $E_{\mathrm{th}}=0.41 \mathrm{eV}, S=10^{12} \mathrm{~s}^{-1}$ and $\tau_{\mathrm{rec}}=0.5 \mathrm{~ns}$. The experimental results are plotted in (b) for comparison. $V_{\mathrm{GS}}$ is $-0.40 \mathrm{~V}$ in (a) and $-0.65 \mathrm{~V}$ in (b) for the top curves, and the potential step is $\Delta V_{\mathrm{GS}}=-0.1 \mathrm{~V}$.

behavior is the opposite to that found for lattice-matched InGaAs/InAlAs HEMTs, in which the increase of $I_{\mathrm{D}}$ is lower for higher $V_{\mathrm{GS}}[8]$. For high $V_{\mathrm{DS}}$ and $V_{\mathrm{GS}}$, although the electron concentration is larger than for low $V_{\mathrm{GS}}$ due to the opening of the channel, the maximum electron energy is reduced due to the lower gate-to-drain potential $[8,9]$. In the case of InAs/AlSb HEMTs, the bandgap of the channel material is much smaller than in InGaAs/ InAlAs HEMTs (and thus the threshold energy $E_{\mathrm{th}}$ ), so that impact ionization probability remains significant even for the higher values of $V_{\mathrm{GS}}$. Concerning the gate current $I_{\mathrm{G}}$, in the experimental device it still shows the typical bell-shape due to the outflow of holes [5], signature of impact ionization in standard Schottky-gate field effect transistors (FETs), in spite of the isolated gate. However, in the ideal simulated structures $I_{\mathrm{G}}$ is zero (the gate current due to hole tunneling is not considered in the MC model).

MC simulations provide an insight into the physical origin of the kink effect in InAs/AlSb HEMTs. In order to analyze the influence of $V_{\mathrm{GS}}$, Fig. 3 shows the profiles along the transistor of: (a) the number of impact ionization events taking place in the channel, (b) the sheet hole density in the buffer, and (c) the sheet electron density in the channel, for $V_{\mathrm{DS}}=0.4 \mathrm{~V}$ and different values of $V_{\mathrm{GS}}$. In Fig. 3c the profiles in the absence impact ionization have been added for comparison. As observed in Fig. 3a, the impact ionization events take place in the drain side of the channel, mainly close to the drain edge of the recess, where the electric field, and consequently the electron energy, is higher. 


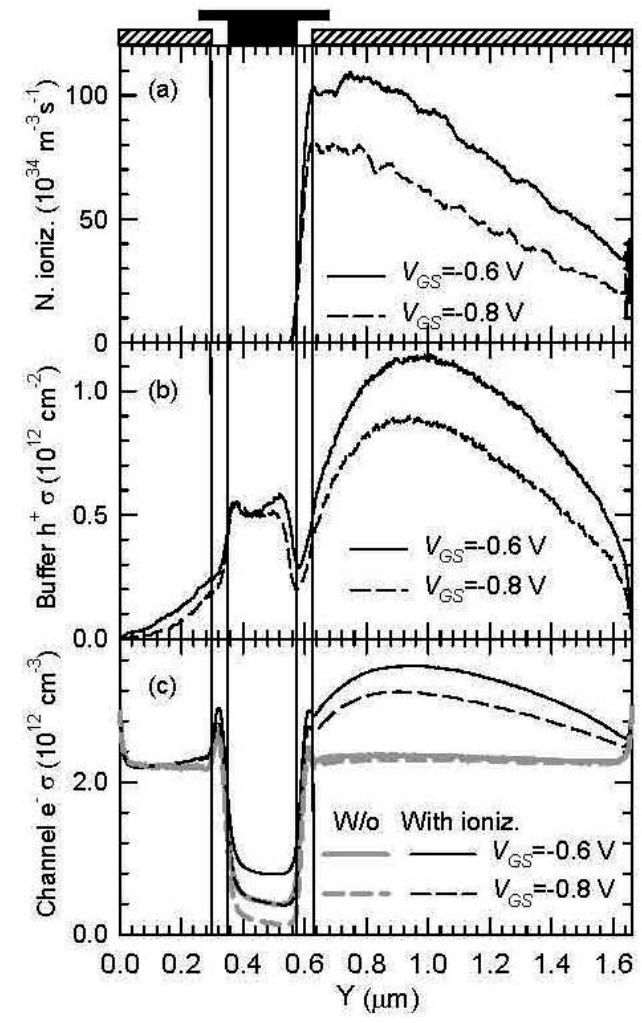

Fig. 3. Profiles along the $Y$ direction of: (a) impact ionization events in the channel per unit time and depth, (b) sheet hole density in the buffer, and (c) sheet electron density in the channel, for different values of $V_{\mathrm{GS}}$, being $V_{\mathrm{DS}}=0.4 \mathrm{~V}, S=10^{12} \mathrm{~s}^{-1}, E_{\mathrm{th}}=0.41 \mathrm{~V}$ and $\tau_{\text {rec }}=0.5$ ns. In (c) the case of absence of impact ionization is included for comparison. The position of the gate and the recess is indicated.

Most of the holes generated by impact ionization in the channel descend the energy step present in the valence band at the heterojunction between the InAs channel and the AlSb buffer. Once in the buffer, the energy of holes decreases because of scattering mechanisms, so that the attracting force of the gate potential and the negative surface charge at the recess is not sufficiently strong for them to surmount the energy barrier in the valence band and return back to the channel. Thus, holes accumulate in the buffer, mainly at the drain side and under the gate contact (Fig. 3b). Few holes reach the source contact because they recombine before arriving at this electrode. The number of electrons/holes generated by impact ionization is very low so as to provide a significant contribution to $I_{\mathrm{D}}$. Indeed, the increase of $I_{\mathrm{D}}$ can be explained just as a consequence of the pile up of positive charge in the buffer, which lowers the potential barrier that controls the current through the channel. As a result, the channel is further opened and the electron density (Fig. 3c) under the gate electrode, and mainly in the drain side of the channel, increases (with the consequent increase of the drain conductance). This leads to the growth of $I_{\mathrm{D}}$ observed in Fig. 2 .

\section{Conclusion}

We have presented a microscopic analysis, based on MC simulations, of kink effect in insulated-gate recessed InAs/AlSb HEMTs. The results allow us interpreting the effect in terms of the pile up of holes (generated by impact ionization at the drain region of the channel) at the drain-gate side of the buffer. This hole pile-up takes place in the buffer because of the energy barrier in the valence band at the heterostructure between the AlSb buffer and the InAs channel. This accumulation of positive charge contributes to further open the channel, thus increasing the electron density in the active part of the device. This leads to a further increase of the drain conductance and the kink effect in the $I-V$ curves.

\section{Acknowledgments}

This work has been partially supported by the European Commission through the ROOTHz project ICT-2009-243845, by the Dirección General de Investigación (MEC) and FEDER through project TEC2007-61259/ MIC and by the Consejería de Educación, Junta de Castilla y León through projects SA019A08 and GR270.

\section{References}

[1] C.R. Bolognesi, M.W. Dvorak, D.H. Chow, IEEE Trans. Electron Dev. 46, 826 (1999).

[2] W. Kruppa, J.B. Boos, B.R. Bennett, N.A. Papanicolaou, D. Park, Robert Bass, IEEE Trans. Electron Dev. 54, 1193 (2007).

[3] Y.C. Chou, M.D. Lange, B.R. Brennett, J.B. Boos, J.M. Yang, N.A. Papanicolau, C.H. Lim, IEEE Electron Dev. Lett. 28, 856 (2007).

[4] M. Malmkvist, E. Lefebvre, M. Borg, L. Desplanque, X. Wallart, G. Dambrine, S. Bollaert, J. Grahn, IEEE Trans. Microwave Theory Tech. 56, 2685 (2008).

[5] E. Lefebvre, M. Malmkvist, M. Borg, L. Desplanque, X. Wallar, G. Dambrine, S. Bollaert, J. Grahn, IEEE Trans. Electron Dev. 56, 1904 (2009).

[6] J. Mateos, T. González, D. Pardo, V. Hoel, H. Happy, A. Cappy, IEEE Trans. Electron Dev. 47, 1950 (2000).

[7] H. Rodilla, T. González, D. Pardo, J. Mateos, J. Appl. Phys. 105, 113705 (2009).

[8] B.G. Vasallo, J. Mateos, D. Pardo, T. González, J. Appl. Phys. 94, 4096 (2003).

[9] B.G. Vasallo, J. Mateos, D. Pardo, T. González, J. Appl. Phys. 95, 8271 (2004).

[10] M.V. Fischetti, IEEE Trans. Electron Dev. 38, 634 (1991).

[11] T. Brudevoll, T.A. Fjeldly, J. Baek, M.S. Shur, J. Appl. Phys. 67, 7373 (1990).

[12] M. Costato, L. Reggiani, Phys. Status Solidi B 58, 471 (1973).

[13] O. Madelung, Semiconductors: Data Handbook, Springer, Berlin 2004.

[14] S. Babiker, A. Asenov, N. Cameron, S.P. Beaumont, IEEE Trans. Electron Dev. 43, 2032 (1996). 